\title{
A Novel Cross-Layer QoS Routing Algorithm for Wireless Mesh Networks
}

\author{
Chi Harold Liu, Kin K. Leung, and Athanasios Gkelias \\ Department of Electrical and Electronic Engineering, Imperial College \\ Exhibition Road, London, SW7 2BT, United Kingdom \\ \{chi.liu06,kin.leung, a.gkelias\}eimperial.ac.uk
}

\begin{abstract}
Wireless mesh networks (WMNs) are expected to support various types of applications with different quality of service $(\mathrm{QoS})$ requirements. Existing works are limited to layered approaches that overlook the interaction between medium access control (MAC) and routing algorithms and often fail to satisfy these requirements in such dynamic wireless environments. The inefficiency of current layered schemes to guarantee these demands has recently triggered the interest for new cross-layered approaches. In this paper, we propose a distributed, multiconstrain, cross-layer QoS routing algorithm for wireless mesh networks that can simultaneous satisfy multiple QoS requirements. Studies with different scheduling algorithms and routing protocols have shown that our algorithm successfully guarantees various QoS requirements and achieves higher network throughput when compared with other standard techniques.
\end{abstract}

\section{INTRODUCTION}

The ongoing proliferation of wireless broadband data services is expected to lead to increased needs on the side of the backhaul network, where the typical upgrade of wired lines to high-speed fibre networks is not always an available or economically attractive solution. In such cases, multihop wireless mesh networks (WMNs) [1], transporting data between the access network and the wired Internet, could offer an appealing alternative. WMN is comprised of wireless mesh routers (WMRs) and wireless access points (APs) (Fig. 1). WMRs form a mesh of self-configuring, self healing links among themselves. With gateway functionality, some of them can be connected to the Internet (Internet gateway: IGW). Unlike classic ad-hoc networks, the transceivers in WMNs do not have energy constrains while most of the applications are broadband services with heterogeneous QoS constraints, including high throughput and low delay, jitter and packet-error-rate (PER). Unfortunately, the traditional protocol layered approach that designs and optimizes the operations of each network protocol layer usually fails to provide the optimal design of wireless multi-hop networks. This is mainly because the dynamic nature of the wireless channel (in terms of capacity, bit error rate etc.) does not guarantee a fixed capacity or reliable connectivity. Hence, in order to provide satisfactory network performance in terms of end-to-end (ETE) quality of service (QoS), cross-layer design becomes crucial. However, to the best of our knowledge, limited research work has been done so far within the field of cross-layer QoS routing and scheduling algorithms design for wireless mesh network. The challenge is twofold. First, QoS routing with

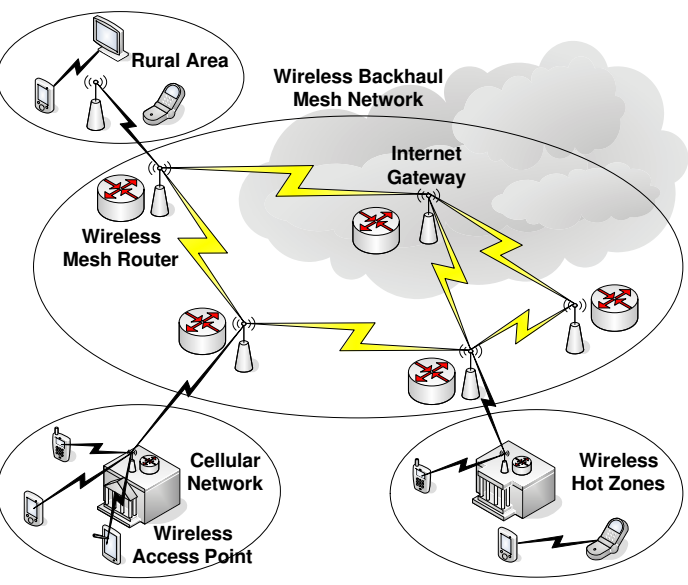

Fig. 1. Typical wireless mesh network scenario.

multiple constraints has been mathematically proven to be NPcomplete by Wang et al. in [2], which shows that the problem of finding a route subject to constraints on two or more additive (delay, jitter) and multiplicative metrics (PER) in any possible combination is NP-complete. Second, scheduling and routing algorithms have different operation time-scales. These become the major challenges to improving protocol efficiencies.

\section{RELATED WORK}

Much work [3]-[4] and has so far studied extensively on multi-constrained QoS routing algorithms in wired network based on network state [5]-[6]. But they cannot been directly applied to wireless mesh networks because unlike the wired network, the network topology may change constantly, the available state information for routing is inherently imprecise, and central control may not exist. On the other hand, multi-constrained QoS routing algorithms for wireless adhoc networks have been previously explored; for instance, QoS-AODV [7] provides QoS provisioning in terms of both bandwidth and delay. However, it overlooks the queueing delay since only the packet processing time was considered. This results in underestimation of the ETE packet delay, especially in case of high traffic load. Another bandwidth routing (BR) protocol [8] and a similar on-demand QoS routing (OQR) protocol [9] were proposed to calculate the available bandwidth in terms of slot reserved for QoS flows. However, because slots are pre-determined before traffic flows are scheduled, it 
fails to exploit the opportunistic scheduling gain in fast-fading channels. In other words, the reserved time slots may lead to poor transmission quality due to bad instantaneous channel conditions. Moreover, [10] addresses both time and reliability constraints, but the average link delay and reliability used to make routing decisions is not able to quickly adapt to fast fading wireless channels. The impact of performance metrics on a routing protocol is studied in [11] where it uses the loss rate of broadcasting packets and round trip delay to select path. However, none of these works explicitly consider MAC/PHY layer parameters thus cannot guarantee QoS.

On the other hand, scheduling for wireless mesh networks has drawn a lot of research attention recently. Due to the fact [12] and [13] that finding a perfect match with the highest network throughput is NP-complete [14]-[15] for centralized scheduling algorithms, various distributed scheduling algorithms have been proposed. Recently, [16]-[17] propose a distributed opportunistic scheduling algorithm for backhaul networks, which provides multi-user diversity gain in the wireless environments, enforces resource allocation in the long run and maintains strong temporal correlation for interference, without which channel quality and interference cannot be tracked and predicted with reasonable accuracy.

In this paper, we propose a novel on-demand, fully distributed, cross-layer integrated QoS routing algorithm (IQoSR). The main contributions are of two folds. First, by formulating a novel integrated QoS performance metric, various QoS constraints are explicitly considered. Second, we propose a cross-layer QoS routing algorithm interacting with distributed opportunistic scheduler in [16] and [17] to optimize the network performance. To the best of our knowledge, this is the first paper not only explicitly considers with multiple performance metrics, but also takes advantage of channel conditions explored by MAC scheduler and therefore proposes a crosslayer scheduling and routing solution. Extensive simulation studies show that our algorithm can successfully guarantee QoS while achieving the best network performance compared with other standard techniques. The remainder of the paper is organized as follows. In Section III, a novel integrated QoS performance metric is introduced. Section IV describes the proposed IQoSR discovery procedures in detail. Extensive simulation results are shown in Section V and Section VI concludes the paper.

\section{Multiple QoS Performance Metrics}

Consider a wireless mesh network comprises a set of $n_{r}$ number of wireless mesh routers, denoted as $V_{R}=\left\{v_{r} \mid r=\right.$ $\left.1,2, \ldots, n_{r}\right\}$ and a set of $n_{g}$ number of Internet Gateways denoted as $V_{G}=\left\{v_{g} \mid g=1,2, \ldots, n_{g}\right\}$. Each WMR independently generates data sessions/flows. Each QoS flow with flow index $q$ has to fulfil a set of QoS constraints that includes endto-end (ETE) packet delay $D_{q}^{r}$, throughput $T_{q}^{r}$ and PER $E_{q}^{r}$. We denote this set as $\left(D_{q}^{r}, T_{q}^{r}, E_{q}^{r}\right)$. A route $\Omega_{s t}^{k}$ from a source WMR with index $s$ to a destination IGW indexed $t$ within the route set $\Omega_{s t}$ is concatenated by a set of links $\left\{\left(v_{i}, v_{j}\right)\right\}$, for all $v_{i}, v_{j} \in V_{R} \bigcup V_{G}$. Therefore, we could formally express the route from $s$ to $t$ as (1) where the total $m$ candidate routes exist. In the following discussions, we use term session and flow for the traffic input, $\left(v_{i}, v_{j}\right)$ and $(i, j)$ for the link between $v_{i}$ and $v_{j}$ interchangeably. For the $k^{t h}$ route,

$$
\Omega_{s t}^{k}=\left\{\left(v_{i}, v_{j}\right) \mid \forall v_{i}, v_{j} \in V_{R} \bigcup V_{G}\right\}
$$

where $k=1,2, \ldots, m$. Let $\Omega_{s t}$ further denote the route set from source mesh router $s$ to a particular gateway node $t$.

In order to find the optimum route that satisfies multiple QoS constrains, we define a "dissatisfaction ratio" $\mathcal{R}$ for each of the QoS requirement as the ratio between expected metric values and value defined by the QoS requirements. For instance, the ETE packet delay dissatisfaction ratio $\mathcal{R}_{k}^{D}$ for route $\Omega_{s t}^{k}$ is defined as the actual delay measurement over the delay requirement. On the other hand, throughput dissatisfaction ratio is formulated as the ratio between the throughput requirement $T_{q}^{r}$ and actual bottleneck link throughput, the minimum of all link throughputs along route $\Omega_{s t}^{k}$. Finally, the dissatisfaction ratio for the PER, $\mathcal{R}_{k}^{E}$, is defined as the multiplication of all one-hop PER over PER requirement $E_{q}^{r}$ since this is a multiplicative constrain. Since a session has to fulfil the set of QoS requirements, a source-to-gateway route will be feasible if and only if all defined ratios of relevant constraints are less than one. In order to efficiently cope with above-mentioned coexisting QoS flows with different relevant requirements in terms of delay, throughput and PER, we introduce the indication function, $\mathcal{I}_{D}, \mathcal{I}_{T}$ and $\mathcal{I}_{E}$ for each QoS constrains, where $\mathcal{I}_{a}$ is denoted as 1 if and only if parameter $a$ is critical for the incoming QoS flow, elsewhere 0, as formally expressed as (2).

$$
\mathcal{I}_{a}= \begin{cases}1 & \text { if requirment } a \text { is required for flow } q \\ 0 & \text { elsewhere }\end{cases}
$$

Moreover, in finding the optimal route for different sessions, we introduce another set of resource reservation factors $\beta_{D}$, $\beta_{T}$ and $\beta_{E}$ for delay, throughput and PER respectively. These not only leave margins for resource reservation in scheduling part to guarantee QoS, but also provide a cross-layer resource allocation interfaces between Network and MAC layers. Hence, we have the utility functions for the delay, throughput and PER as, $U_{k}^{D}(q)=\mathcal{I}_{D} \mathcal{R}_{k}^{D}(q), U_{k}^{T}(q)=\mathcal{I}_{T} \mathcal{R}_{k}^{T}(q)$ and $U_{k}^{E}(q)=\mathcal{I}_{E} \mathcal{R}_{k}^{E}(q)$ in route $\Omega_{s t}^{k}$. With (2)-(3), a multiobjective function is introduced in order to find the optimal route for session $q$ in a heuristic way by taking into account multiple QoS constraints simultaneously.

$$
\begin{aligned}
& \min _{\forall \Omega_{s t}^{k} \in \Omega_{s t}} \max \left[U_{k}^{D}(q), U_{k}^{T}(q), U_{k}^{E}(q)\right] \\
= & \min _{\forall \Omega_{s t}^{k} \in \Omega_{s t}} \max \left[\mathcal{I}_{D} \mathcal{R}_{k}^{D}(q), \mathcal{I}_{T} \mathcal{R}_{k}^{T}(q), \mathcal{I}_{E} \mathcal{R}_{k}^{E}(q)\right]
\end{aligned}
$$

Subject to :

$$
\left\{\begin{array}{l}
\mathcal{R}_{k}^{D}(q)=\frac{\sum_{(i, j) \in \Omega_{s t}^{k}} D_{i j}^{a}}{\left(1-\beta_{D}\right) D_{q}^{r}} \leq 1 \\
\mathcal{R}_{k}^{T}(q)=\frac{\left(1+\beta_{T}\right) T_{q}^{q}}{\min _{(i, j) \in \Omega_{s t}^{k} T_{i j}^{a}} \leq 1} \\
\mathcal{R}_{k}^{E}(q)=\frac{1-\prod_{(i, j) \in \Omega_{s t}^{k}}\left(1-E_{i j}^{a}\right)}{\left(1-\beta_{E}\right) E_{q}^{r}} \leq 1
\end{array}\right.
$$




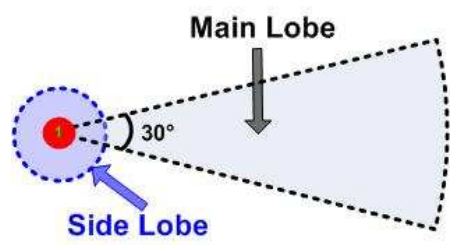

Fig. 2. An example of directional antenna pattern used in cross-layer simulation with side lobe gain $-25 \mathrm{~dB}$ and main lobe sector in $30^{\circ}$.

The above distributed optimization function runs on each node, where "min-max" operator takes the minimum dissatisfactory ratio among the set of all possible routes $\Omega_{s t}$ from the source WMR $s \in V_{R}$ to a certain IGW $t \in V_{G}$.

\section{Proposed IQoS Routing Procedure}

Assuming fixed transmission power in each WMR and certain signal receiving threshold, each mesh router maintains its immediate neighboring nodes. We also assume the timedivision duplex (TDD) system is used where each link can only transmit or receive signal but not both at any given time, is considered. By using directional antenna techniques, as shown in Fig. 2, link quality/capacity is greatly improved due to the decrease of interferences. Thus, network throughput is improved and more traffic load could be routed.

Routing discovery phase requires each receiving of each link to record one-hop delay, link throughput and PER information, which are to be used later. By introducing an example of routing discovery procedures in Fig. 3, we show how our integrated QoS routing discovery phase works.

Routing discovery procedure is initialized when new traffic flows are accepted by certain nodes. As an example, in Fig. 3 , at given time WMR 1 serves as the source. It generates a request packet $R E Q$ containing the QoS flow constraints and starts a timer when sending the $R E Q$ through the allocated time slot in the control channel to its one-hop neighbors. Before the timer expires, if WMR 1 does not receive a reply message $R E P$, it will regenerate a request packet and broadcasts it to the whole network due to possible packet loss. In Fig. 3(a), when node 2 receives $R E Q$, it averages previous one-hop delay $D_{12}^{a}$, link throughput $T_{12}^{a}$ and PER $E_{12}^{a}$ measurements, which were recorded in mesh router 2 , then piggybacks this information in $R E Q$ and sends it to WMR 1, WMR 3 and WMR 6 through the allocated time slots in the control channel. Nevertheless, only node 3 and node 6 need to forward this message to their neighbours after piggybacking $D_{i j}^{a}, T_{i j}^{a}$ and $E_{i j}^{a}$ for the corresponding link $\left(v_{i}, v_{j}\right)$ in the $R E Q$ packet.

All other nodes in the network repeat these procedures until gateway node 5 receives the request message. Then, the reply procedure is initialized in Fig. 3(b). It sends a reply packet REP back through two different routes to mesh router 1: $5 \rightarrow 4 \rightarrow 3 \rightarrow 2 \rightarrow 1$ and $5 \rightarrow 4 \rightarrow 6 \rightarrow 7 \rightarrow 2 \rightarrow 1$. By calculating the integrated QoS performance metric using (3), WMR 1 chooses the best route obtained before the timer expires. It is also worth noting that WMR 1 does not have to wait for all reply messages, not only because the large number of possible routes (even for reasonable network size), but also

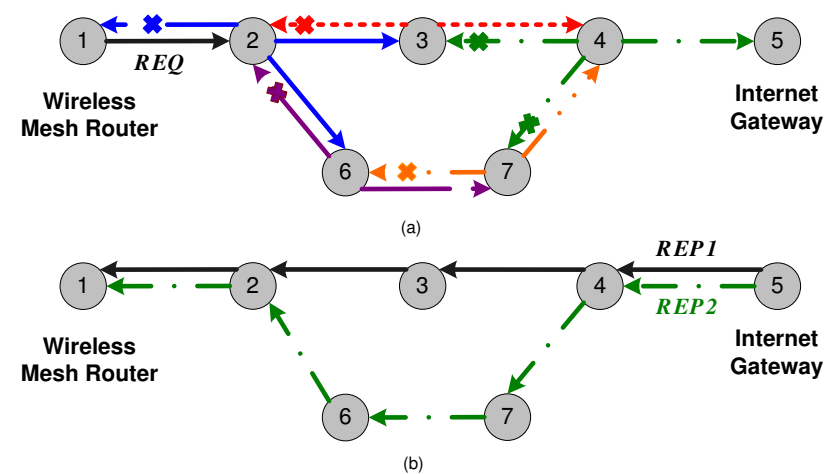

Fig. 3. An example of proposed IQoS routing algorithm discovery procedures. (a) REQ packets broadcasting to neighbors (b) Gateway nodes send REP packets back to source.

because we rather need to meet the certain QoS requirements rather than finding the best route (which may generate huge overhead).

\section{NUMERICAL RESUlts}

We develop a slotted, time-driven simulator using MATLAB and $\mathrm{C}++$ which comprises physical (PHY), MAC and Network Layers, where channel model/adaptive modulation and coding schemes, different MAC scheduling and routing algorithms are implemented respectively. WMRs and IGWs are randomly deployed in 2D-square in a way that no disconnected clusters of nodes exist in the network. Data traffic is generated according to Poisson process with inter-arrival time in terms of slots for each WMR to be routed to certain IGWs. Different traffic patterns are considered, with three QoS constraints, i.e., throughput, ETE packet delay and PER. ETE packet delay consists of queuing, transmission and processing delays. In PHY layer, the Jake's Model [18] is used to generate the link characteristics among WMRs and IGWs. PER is simulated based on the signal-to-interference-plus-noise-ratio (SINR) curve for the used adaptive modulation and coding scheme (AMC). At given time $t$, receiving SINR $\gamma_{i j}$ for link $\left(v_{i}, v_{j}\right)$ is calculated as,

$$
\gamma_{i j}=\frac{P_{i j}^{T x} C_{i j} d_{i j}^{-\alpha}}{\sum_{k} P_{k j}^{T x} C_{k j} d_{k j}^{-\alpha}+\mathcal{N}_{0}}
$$

where $P_{i j}^{T x}, C_{i j}$ and $d_{i j}^{-\alpha}$ are transmission power, channel gain (the antenna gain has been also included here) and path loss between link $\left(v_{i}, v_{j}\right)$ respectively. Typical value for path loss coefficient $\alpha$ is 3.5. $\mathcal{N}_{0}$ is the single-sided power spectrum density for additive white Gaussian noise. Retransmission scheme is assumed in case of packet loss. Each WMR is equipped with directional antenna and assume accurate positioning.

The performance of our proposed routing algorithm is evaluated in terms the proposed QoS performance metrics, namely, achieved throughput, end-to-end packet delay and PER. Table I summarizes the simulation parameters and Fig. 4(a) shows a typical wireless mesh network scenario and node 
(a)

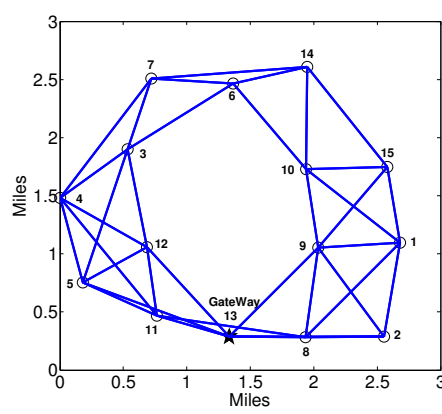

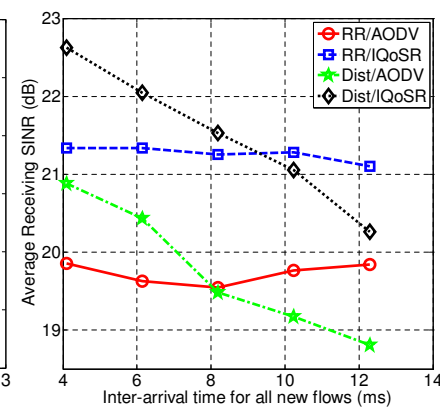

(b)
Fig. 4. (a) An example to show a typical simulation topology with node connectivity, where there are 15 wireless mesh routers and one of them is chosen to be Internet gateway node (b) Performance evaluation on average receiving SINR $\gamma$ w.r.t. traffic load.

TABLE I

SiMULATION PARAMETERS

\begin{tabular}{l|l|l|l}
\hline \hline Parameter & Value & Parameter & Value \\
\hline Channel Model & Jakes Model & Path Loss Coeff. & $2-4$ \\
\hline Antenna Pattern & $\begin{array}{l}\text { Side lobe: }-25 \mathrm{~dB} \\
\text { Main lobe: } 30^{\circ}\end{array}$ & AMC & $\begin{array}{l}\text { MPSK } \\
\text { MQAM }\end{array}$ \\
\hline Doppler Freq. & $10-25 \mathrm{~Hz}$ & System Bandwidth & $50 \mathrm{MHz}$ \\
\hline Slot Duration & $0.04 \mathrm{~ms}$ & Slots per Frame & 160 \\
\hline Frame Duration & $6.4 \mathrm{~ms}$ & Packet Length & 512 bytes \\
\hline WMR & 15 & IGW & 1 \\
\hline Network Size & $3 \mathrm{miles}$ & Tx Range & 1.5 miles \\
\hline Traffic Arrival & Poisson & & \\
\hline \hline
\end{tabular}

connectivity with 15 wireless mesh routers and one router chosen as the gateway.

To assess the performance of the proposed IQoSR algorithm with distributed opportunistic scheduler, we compare it with three other schemes by using Fair Round Robin scheduler [19] and AODV [20] routing protocol as our benchmark. Table II summarizes these four comparisons. Fig. 5 shows the overall network performance in terms of average integrated QoS performance metric per-packet based value calculated by (3) and gateway/network throughput, with respect to traffic inter-arrival time. Furthermore, ETE packet delay, throughput and PER average values and satisfactory ratios are plotted in Fig. 5 to the efficiencies of our algorithms.

Metric values smaller than one in Fig. 5(a) are considered as packets with all QoS guarantees. While decreasing traffic load, this metric value for all four comparisons drop in different scales, because fewer packets are injected into the network and therefore less queuing delay, higher packet throughput could be expected in Fig. 5(c) and Fig. 5(e). However, only the

TABLE II

FOUR COMPARISONS

\begin{tabular}{c|c|c|c}
\hline \hline & MAC Scheduling & Routing & Cross-Layer Term \\
\hline 1 & Fair Round Robin & AODV & RR/AODV \\
\hline 2 & Fair Round Robin & IQoSR & RR/IQoSR \\
\hline 3 & Distributed Opportunistic & AODV & Dist/AODV \\
\hline 4 & Distributed Opportunistic & IQoSR & Dist/IQoSR \\
\hline \hline
\end{tabular}

distributed opportunistic scheduler with IQoSR successfully achieves the average QoS performance metric value less than 1 after traffic inter-arrival time is higher than $6 \mathrm{~ms}$. This is because the pre-determined nature of the round robin scheduler does not take the advantage of the multi-user diversity gain of fading channels; and AODV routing protocol fails to guarantees QoS constraints and thus all new traffics are admitted regardless of network situation. Nevertheless, the distributed opportunistic scheduler provides multi-user diversity gain both in time and space domains by exploring channel conditions while minimizing total interference. Meanwhile, the IQoSR algorithm with admission control policy guarantees various QoS requirements and thus better network overall performances could be expected. This could be seen from Fig. 5(b) which shows Dist/IQoSR combination achieves highest gateway/network throughput, even $600 \%$ more packets received than RR/AODV combination, $300 \%$ and $130 \%$ more than RR/IQoS and Dist/AODV combinations respectively, when traffic load is very high. Similar trends can be seen in all these figures with a large throughput increase, the delay and PER decrease if using the Dist/IQoSR combination.

However, it is also worth noting that although PER curves with respect to traffic load in Fig. 5(g) for both Dist/IQoSR and Dist/AODV combinations are always better than the other two using round robin scheduler, it also shows the increase of PER values and decrease of satisfactory ratios. This is because that distributed opportunistic scheduler loses multiuser diversity gain when traffic load is relatively low. In other words, this scheduler is not smart enough to stop scheduling packet transmissions when channel quality is poor, thus lower receiving SINR trend could also be expected in Fig. 4(b). Nevertheless, the PER satisfactory ratio for the Dist/IQoSR combination is always higher than the others, which means that an improved chance for our algorithm to successfully meet the PER requirements. Furthermore, Fig. 4(b) also shows that our routing algorithm roughly has $1.5 \mathrm{~dB}$ SINR gain if the fixed scheduling is used.

\section{Vi. Conclusions}

In this paper, a novel on-demand, fully distributed crosslayer integrated QoS routing algorithm has been proposed. Extensive simulation results show that a combination of the distributed opportunistic scheduler with the IQoSR algorithm achieves higher network performance gain when compared to other combinations such as round-robin scheduler and AODV routing protocol. Moreover, the proposed integrated QoS performance metrics can be easily extended to other metrics such as delay jitter or user-defined link utilities, and used for multi-path routing. The next stage of our research will include the extension of this framework to incorporate MIMO antennas to further improve the network performance in non-line-of-sight (NLOS) environments. 


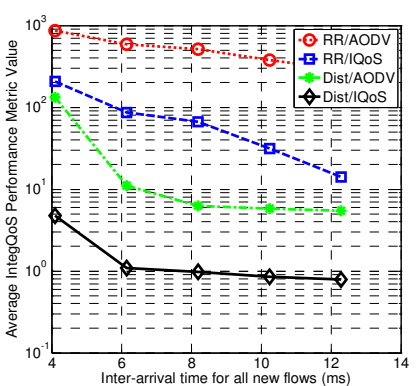

(a)

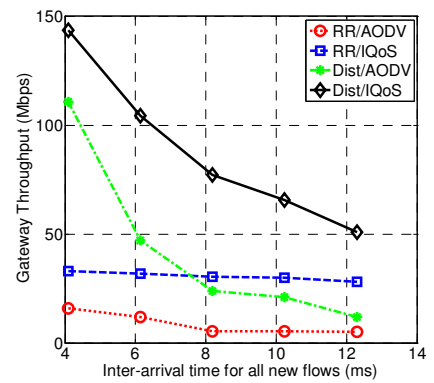

(b)

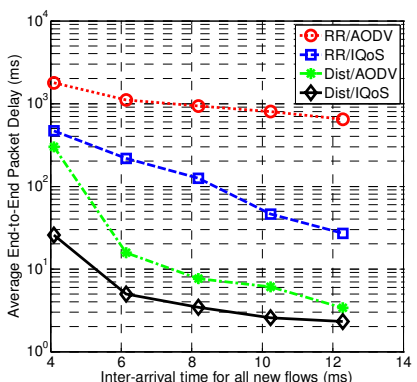

(c)

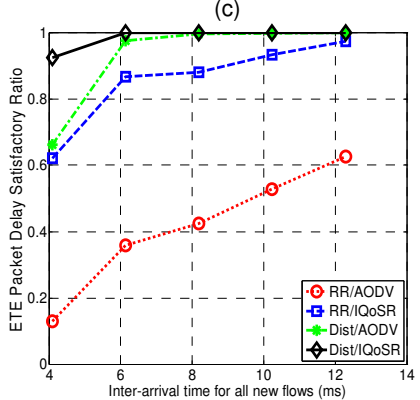

(d)

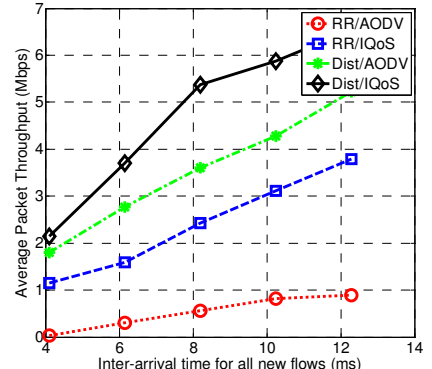

(e)

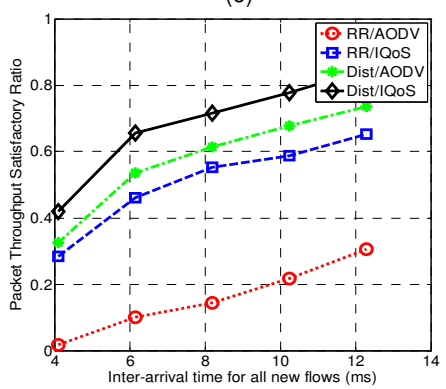

(f)

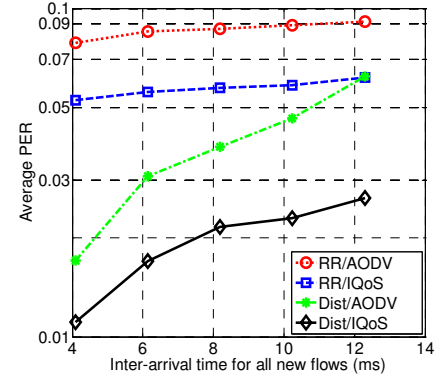

(g)

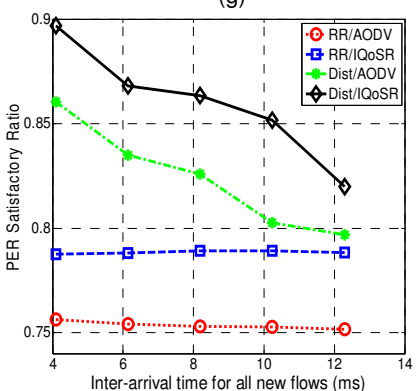

(h)

Fig. 5. The effect of network traffic on (a) Average Integrated QoS performance metric value (b) Gateway/network throughput (c) Average ETE packed delay (d) Packet delay satisfactory ratio (e) Average packet throughput (f) Packet throughput satisfactory ratio (g) Average PER (h) PER satisfactory ratio. All w.r.t. traffic load.

\section{ACKNOWLEDGMENT}

This research was financially supported by grants from EU IST FP6 MEMBRANE project under the contracts 027310.

\section{REFERENCES}

[1] I. F. Akyildiz and X. Wang, "A survey on wireless mesh networks," IEEE Communications Magazine, vol. 43(9), pp. S23-S30, Sept. 2005.

[2] Z. Wang and J. Crowcroft, "Quality-of-service routing for supporting multimedia applications," IEEE JSAC, vol. 14(7), pp. 1228-1234, Sept. 1996.

[3] X. Yuan, "Heuristic algorithms for multiconstrained quality-ofservice routing," IEEE/ACM Trans. on Networking, vol. 10(2), pp. 244-256, Apr. 2002.

[4] J. M. Jaffe, "Algorithms for finding paths with multiple constraints," IEEE Networks, vol. 14, pp. 95-116, Apr. 1984.

[5] P. V. Mieghem and F. A. Kuipers, "On the complexity of qos routing," Computer Communications, vol. 26(4), pp. 376-387, Mar. 2003.

[6] T. Korkmaz and M. Krunz, "Bandwidth-delay constrained path selection under inaccurate state information," IEEE/ACM Trans. on Networking, vol. 11(3), pp. 384-398, Jun. 2003.

[7] Y. Zhang and T. Gulliver, "Quality of service for ad hoc on-demand distance vector routing," in Proceedings of WiMob'2005, vol. 3, Aug. 2005, pp. 192-196.

[8] C. R. Lin and J. Liu, "Qos routing in ad hoc wireless networks," IEEE JSAC, vol. 17(8), pp. 1426-1438, 1999.

[9] C. R. Lin, "On-demand qos routing in multihop mobile networks," in Proceedings of IEEE INFOCOM 2001, vol. 3, Apr. 2001, pp. 17351744.

[10] E. Felemban, C. G. Lee, R. Boder, and S. Vural, "Probabilistic qos guarantee in reliability and timeliness domains in wireless sensor networks," in Proceedings of IEEE INFOCOM 2005, Mar. 2005.

11] R. Draves, J. Padhye, and B. Zill, "Comparisons of routing metrics for static multi-hop wireless networks," in Proceedings of ACM Annual Conf. Special Interest Group on Data Communication (SIGCOMM), Aug. 2004, pp. 133-144.

[12] L. Chen, S. H. Low, J. C. Doyle, and M. Chiang, "Cross-layer congestion control, routing and scheduling design in ad hoc wireless networks," in Proceedings of IEEE INFOCOM 2006, Barcelona, Spain, 2006.
[13] S. Ramanathan, "Scheduling algorithms for multihop radio networks," IEEE/ACM Transactions on Networking, vol. 1(2), pp. 166-177, Sept. 1993.

[14] L. Lovasz, Matching theory. North-Holland, 1986.

[15] M. R. Garey, Computers and intractability: a guide to the theory of NP-completeness. W. H. Freeman, 1979.

[16] Y. Hou and K. K. Leung, "Framework of opportunistic allocation of wireless resources," in Proceedings of PacRim 2007, Victoria, B.C., Canada, Aug. 2007.

[17] _ _ "A novel distributed scheduling algorithm for mesh networks," in Proceedings of IEEE Globecom 2007, U.S.A., 2007.

[18] W. C. Jakes, Microwave Mobile Communications, New York. Wiley, 1974.

[19] X. Yuan and Z. Duan, "FRR: a proportional and worst-case fair round robin scheduler," in Proceedings of IEEE INFOCOM 2005, vol. 2, U.S.A., 2005, pp. 831-842.

[20] C. Perkins and E. Royer, "Ad-hoc on-demand distance vector routing," in Proceedings of WMCSA'99, San Jose, CA, USA, 1999, pp. 90-100. 